\title{
Study on Glycemic Status and Oxidative Stress Level in Patients of Type 2 Diabetes Mellitus With and Without Retinopathy: A Teaching Hospital Based Study
}

\author{
Rachana Gahlawat ${ }^{1}$, Dinesh Narain Saksena ${ }^{2}$ \\ ${ }^{1}$ Senior Resident, Department of Ophthalmology, World College of Medical Sciences Research and Hospital, Gurawar, Jhajjar, ${ }^{2}$ Assistant Professor, Department of \\ Ophthalmology, World College of Medical Sciences Research and Hospital, Gurawar, Jhajjar.
}

\section{Abstract}

Background: Diabetes is a chronic disease and sustained hyperglycemia attacks both micro vessels and macro vessels throughout the body. It is the leading cause of retinopathy, nephropathy, end-stage renal disease, non traumatic lower extremity amputations. Subjects and Methods: Twenty five (25) Patients with newly diagnosed type 2 diabetes mellitus without any signs of retinopathy were identified from outpatient services of department of Medicine and Twenty five (25) Patients with newly diagnosed type 2 diabetes mellitus with retinopathy were identified from outpatient services of department of Ophthalmology along with 25 healthy subjects were taken as controls. Diabetic retinopathy was diagnosed after doing detailed dilated fundoscopic examination as per the standard criteria. Results: The levels of FBS, $\mathrm{HbA1c}$, total cholesterol, triglycerides and MDA were significantly higher in the diabetics without retinopathy, while the HDL and Vitamin C levels were lower as compared to those of the control group. The levels of FBS HbA1c, cholesterol, triglycerides and MDA were significantly higher in the diabetics with retinopathy, while the HDL and Vitamin C levels were lower as compared to those in the diabetes without retinopathy. Conclusion: The increased oxidative stress and a decreased antioxidant status can predict the micro-vascular complications in diabetes mellitus. The raised MDA levels indicate the oxidative stress and the decreased Vitamin $\mathrm{C}$ levels indicate the reduced antioxidant status in diabetic retinopathy.

Keywords: Diabetic retinopathy, HbA1c and Oxidative Stress.

Corresponding Author: Dr. Dinesh Narain Saksena, Assistant Professor, Department of Ophthalmology, World College of Medical Sciences Research and Hospital, Gurawar, Jhajjar-124103.

Email: rachanagahlawat9@gmail.com

Received: February 2020

Accepted: February 2020

\section{Introduction}

Diabetes mellitus (DM), a chronic progressive disorder, is the result of body's inability to produce insulin or use insulin to its full potential, and is characterized by high circulating glucose. Diabetes is a chronic disease and sustained hyperglycemia attacks both micro vessels and macro vessels throughout the body. It is the leading cause of retinopathy, nephropathy, end-stage renal disease, non traumatic lower extremity amputations. The prevalence of diabetes for all age-groups worldwide was estimated to be $2.8 \%$ in 2000 and $4.4 \%$ in 2030 . The total number of people with diabetes is projected to rise from 171 million in 2000 to 366 million in 2030.The maximum absolute increase in the number of people with diabetes will be in India. According to WHO the estimated number of diabetic cases in India was 31.7 million which is expected to rise to 79.4 million in 2030. In patients diagnosed with diabetes before the age of 30 years the incidence of DR after 10 years is $50 \%$ and after 30 years is $90 \%$. Of those with onset at 30 years or more, $40 \%$ of who were taking insulin and $24 \%$ who were not had some degree of retinopathy when duration of diabetes was less than 5 years and $84 \%$ of those who were taking insulin and $53 \%$ of those who were not had some degree of retinopathy, when the duration of diabetes Wass 15-20 years. ${ }^{[1,2]}$ Approximately $25 \%$ of patients with diabetes mellitus have been shown to be affected with retinopathy with incidence increasing to $60 \%$ after 5 years and $80 \%$ after 10 to 15 years of affliction. ${ }^{[3]}$ DM is characterized by hyperglycaemia, together with the biochemical alteration of glucose and lipid peroxidation. Oxidative stress is increased in DM, owing to an increase in the production of oxygen free radicals and a deficiency in the antioxidant defense mechanisms. The lipid peroxidation of the cellular structures, a consequence of the increased oxygen free radicals, is thought to play an important role in the atherosclerosis and the micro-vascular complications. ${ }^{[4]}$ Patients with long standing Type 2 DM have a higher prevalence of severe visual impairment which is usually associated with diabetic retinopathy. The incidence of retinopathy which increases with an increasing duration of diabetes and sustained hyperglycaemia has been identified as the major risk factor in the development of this micro-vascular complication. It is known that $20-50 \%$ of 
the long duration diabetes cases show proliferative diabetic retinopathy. This type of retinopathy progresses from mild non-proliferative abnormalities to proliferative retinopathy which is characterized by the growth of new vessels on the retina and on the posteriorsurface of the vitreous. The exact biochemical mechanism whichcauses the initiation and progression of diabetic retinopathy has been poorly understood but still it has been proved that the blood supply to the essential organs including the retina is reduced in longstanding diabetes mellitus, owing to a failure in the auto-regulatory mechanisms. We assume that ischaemia in the inner retinal tissues produces some biochemical changes which are responsible for the morphological changes in the retina. ${ }^{[5]}$ Diabetes duration and sustained hyperglycemia are among the primary risk factors for the development of diabetic retinopathy. ${ }^{[6]}$ The use of Fasting blood sugar and HbA1c testing may help predict those at-risk for diabetes, diabetic retinopathy or other complications of diabetes ${ }^{[7]}$ The study is intended to know whether these exists an involvement in between antioxidant nutrient intake and reduction in the improvement of diabetic complications particularly retinopathy. ${ }^{[8]}$ Supplementary Vitamin C may be helpful in decreasing blood glucose in type 2 diabetes and thus reducing the risk of complications. ${ }^{[9]}$

Oxidative stress is increased in diabetes mellitus, owing to an increased production of free radicals such as the super oxide radical, peroxide and free radical induced lipid per oxidation. Taking into consideration the above facts, that chronic hyperglycaemia leads to the formation of free radicals and free radical induced lipid peroxidation, which cause microangiopathic changes like retinopathy in DM, Hence the present study was undertaken to the role of oxidative stress and its correlation with hyperglycaemia in patients of Type $2 \mathrm{DM}$ with and without retinopathy.

\section{Subjects and Methods}

This present study was conducted in the Department of Ophthalmology, World College of Medical Sciences Research and Hospital, Jhajjar, in collaboration with the Department of Medicine and Biochemistry during the period from February to june, 2019. Twenty five (25) Patients with newly diagnosed type 2 diabetes mellitus without any signs of retinopathy were identified from outpatient services of department of Medicine and Twenty five (25) Patients with newly diagnosed type 2 diabetes mellitus with retinopathy were identified from outpatient services of department of Ophthalmology along with 25 healthy subjects were taken as controls. Diabetic retinopathy was diagnosed after doing detailed dilated fundoscopic examination as per the standard criteria. ${ }^{[10]}$

\section{Biochemical parameters studied:}

An overnight fasting $5 \mathrm{ml}$ of venous blood sample was collected for the estimation of fasting plasma glucose, $\mathrm{HbA} 1 \mathrm{c}$ and lipid profile were studied for following parameters.

1. Fasting Blood Glucose by GOD-POD method.

2. GlycatedHaemoglobin bycation exchange resin method.

3. Total Cholesterol by enzymatic end point CHOD-POD method.

4. Triglyceride by enzymatic glycerol phosphate oxidase/peroxidase method.

5. High Density Lipoprotein-Cholesterol by direct enzymatic end point method.

6. Malondialdehyde by Thiobarbuturic acid method.

7. Vitamin-C by 2,4,dinitrophenyl hydrazine method.

\section{Statistical analysis:}

Statistical analysis of data was performed using the SPSS-16. For comparison of parameters between the diabetic with retinopathy, without retinopathy and normal healthy subjects, we used student t-test and pearson's correlation coefficient to find the statistical significance. A P-value $<0.05$ was to be considered statistically significant.

\section{Results \& Discussion}

A total of 75 patients were included; 25 healthy subjects in group-I, 25 diabetic subjects without retinopathy in group-II and 25 diabetic subjects with retinopathy in group-III. Mean values of all parameters and p-values are given in table-1. The results which were obtained from the controls, diabetics without retinopathy and diabetics with retinopathy are shown in [Table1]. The levels of FBS, HbA1c, total cholesterol, triglycerides and MDA were higher in diabetic patients, while HDL and Vitamin C levels were lower as compared tocontrol group. The levels of FBS, HbA1c, total cholesterol, triglycerides and MDA were higher in diabetics with retinopathy, while the HDL and Vitamin C levels were lower as compared to those in the diabetes without retinopathy.

\begin{tabular}{|c|c|c|c|c|c|c|}
\hline \multirow[t]{2}{*}{ Variables } & \multirow{2}{*}{$\begin{array}{l}\text { HealthySubjects-[Gr.- } \\
\text { I] }(\mathrm{N}=\mathbf{2 5}) \\
\text { Mean } \pm \text { S.D. }\end{array}$} & \multirow{2}{*}{$\begin{array}{l}\text { Without retinopathy- } \\
{[\text { Gr.-II] }](\mathrm{N}=25)} \\
\text { Mean } \pm \text { S.D. }\end{array}$} & \multirow{2}{*}{$\begin{array}{l}\text { Diabetic retinopathy- } \\
\text { [Gr.-III](N=25) } \\
\text { Mean } \pm \text { S.D. } \\
\end{array}$} & \multicolumn{3}{|c|}{$P$ value } \\
\hline & & & & I \& II & I \& III & II \& III \\
\hline Age in Years & $38.4 \pm 5.42$ & $52.6 \pm 6.7$ & $54.1 \pm 8.4$ & 0.01 & 0.01 & $0.42^{*}$ \\
\hline FBS (mg/dl) & $87.59 \pm 20.3$ & $146.9 \pm 54.7$ & $152.3 \pm 44.9$ & 0.001 & 0.001 & $0.82 *$ \\
\hline HbA1c (\%) & $5.9 \pm 0.62$ & $9.04 \pm 1.98$ & $9.8 \pm 2.75$ & 0.001 & 0.001 & $0.32 *$ \\
\hline $\mathrm{TC}(\mathrm{mg} / \mathrm{dl})$ & $167.55 \pm 28.7$ & $178.12 \pm 39.47$ & $182.9 \pm 50.57$ & 0.24 & 0.31 & 0.72 \\
\hline TG (mg/dl) & $133.27 \pm 57.6$ & $154.72 \pm 6.02$ & $167.74 \pm 52.7$ & 0.04 & 0.04 & 0.78 \\
\hline HDL-c (mg/dl) & $46.1 \pm 9.7$ & $38.77 \pm 11.34$ & $38.5 \pm 12.2$ & 0.03 & 0.03 & 0.12 \\
\hline MDA (nmol/dl) & $212.6 \pm 72.32$ & $439.7 \pm 120.3$ & $1039.2 \pm 658.5$ & 0.001 & 0.000 & 0.001 \\
\hline Vitamin-C (mg/dl) & $0.98 \pm 0.3$ & $0.92 \pm 0.29$ & $0.86 \pm 0.27$ & $0.34 *$ & 0.001 & 0.01 \\
\hline
\end{tabular}


The plasma MDA levels were significantly elevated and the Vitamin $\mathrm{C}$ levels were decreased in all the diabetic groups as compared to those in the controls. Correlation of this study revealed a significant positive association of plasma MDA with fasting plasma glucose, $(\mathrm{r}=0.45, \mathrm{p}<0.01$, $)$ and HbA1c $(r=0.31, p<0.01)$, thus suggesting the role of hyperglycaemia in free radical production. The plasma MDA levels also indicated a significant positive relation $(\mathrm{p}<0.001)$ with all the lipid parameters except serum HDL$\mathrm{c}$, thus explaining the role of dyslipidaemia in the liberation of the free radicals. A statistically significant positive correlation was found between HbA1c and MDA $(r=0.12)$ and between the triglycerides and MDA $(r=0.46)$. There was a negative correlation between MDA and Vitamin C ( $\mathrm{r}$ $=-0.02)$ in the cases of diabetic retinopathy. Diabetic retinopathy is the most specific of all the diabetic microvascular complications. The prevalence of retinopathy is related to the duration of diabetes. Diabetic retinopathy is the leading cause of new blindness in persons who are aged 30-74 years. The incidence of retinopathy is rarely detected in the first few years of diabetes, but the incidence increases to $50 \%$ by 10 years and to $90 \%$ by 25 years of having diabetes. The prevalence of diabetic retinopathy is increasing due to the prolonged survival of the diabetic patients. In south India, diabetic retinopathy was detected in $1.78 \%$ of the patients who were screened and it was projected to become a significant cause of blindness in the coming decades. Subjects with a duration of diabetes which was $<5$ years had $22.9 \%$ of diabetic retinopathy, while those with diabetes for $>5$ years had $33.5 \%$ of diabetic retinopathy. Younger subjects ( $<40$ years) had $17.9 \%$ of diabetic retinopathy, while those who were $>40$ years of age had $36 \%$ of diabetic retinopathy. Subjects with HbA1c which was $<7 \%$ had a $12.5 \%$ prevalence as compared to those with $\mathrm{HbA} 1 \mathrm{c}$ which was $>7 \%$, who had a prevalence of $40.5 \% .^{[11]}$ The pathogenesis of DR has not been completely understood, but the established risk factors include poor glycaemic control, hypertension, increasing age and the duration of diabetes. ${ }^{[12]}$ A study on the epidemiology of diabetes complications demonstrated that high triglyceride and high LDL levels at the baseline were associated with the subsequent progression of retinopathy over 2 years. ${ }^{[13]}$ It is found that a poor metabolic control which was demonstrated by high HbA1c levels was directly proportional to the prevalence of DR, which has been documented by Klein et al. in 1988. ${ }^{[14]}$ A longer duration of diabetes has been seen to have a statistically high significant correlation with the prevalence of DR. ${ }^{[14]}$ Oxidative stress plays a major role in the onset of diabetes mellitus, as well as in the development of vascular and neurological complications of the disease. ${ }^{[15]}$ The source of the oxidative stress is a cascade of Reactive Oxygen species (ROS) which leak from the mitochondria. ${ }^{[16]}$ Hyperglycaemia contributes to micro-vascular complications. The prominent biochemical pathways which explain how diabetes causes damage to the micro-vasculature system include: (a) an increased polyol pathway flux (b) the production of advanced glycation end products (AGE) (c) the generation of reactive oxygen species (ROS) and (d) the activation of the diacyl glycerol and the protein kinase $\mathrm{C}$ isoforms. ${ }^{[17,18]}$ AGEs are the products of glycation and oxidation and they are responsible for the liberation of superoxide radicals. Excess glucose enters the polyol pathway, resulting in excess sorbitol production, with a concomitant decrease in the NADPH levels. Low levels of NADPH can decrease nitric oxide production in the endothelial cells and can adversely affect the cellular redox balance, thereby resulting in deleterious metabolic consequences. NADPH is required for regenerating the reduced glutathione and the consumption of NADPH could contribute to an intracellular increase in the formation of the reactive oxygen species, thus leading to oxidative stress and resultant diabetes related vascular damage. Therefore, an increased flux through the polyol pathway can lead to micro-vascular damage by contributing to AGE formation, specific protein kinase $\mathrm{C}$ activation and the generation of reactive oxygen species (ROS). Oxidative stress in hyperglycaemia is amplified by the metabolic stress. ${ }^{[18]}$ In the present study, the production of the oxygen free radicals was directly related to hyperglycaemia and the duration of diabetes. The MDA levels acted as a marker for lipid peroxidation i.e. oxidative stress and it was significantly increased in the cases as compared to the controls. In a state of poor metabolic control, increased serum MDA levels are expected. A positive correlation was found between the mean serum MDA levels and the mean HbA1c levels in the diabetic patients. ${ }^{[19]}$ The longer the duration of the disease, the higher were the lipid peroxide levels. Thus, the increase in the lipid peroxides in blood, coupled with the weakness of the defense antioxidant system in diabetics with complications, probably served as a background for the pathogenesis of the endothelial dysfunction which was associated with diabetes. The retina is highly susceptible to oxidative stress because of its high consumption of oxygen, its proportion of polyunsaturated fatty acids (PUFA) and its exposure to visible light. Studies have consistently shown that photochemical injury is attributable to oxidative stress and that the antioxidants, Vitamins $\mathrm{A}, \mathrm{E}$ and $\mathrm{C}$ protect against this type of injury. ${ }^{[20]}$ The levels of Vitamin C, which is a measure of the antioxidant status, is significantly decreased in diabetic patients. In the present study, HbA1c was significantly elevated in diabetic patients, which indicated their glucose status for the past 3 months. There was a significant elevation of triglycerides in patients with complications, indicating dyslipidaemia.

\section{Conclusion}

These findings suggest that, the poor glycaemic control, the long duration of diabetes and dyslipidaemia contributed to the increased oxidative stress. The increased oxidative stress and a decreased antioxidant status can predict the micro-vascular complications in diabetes mellitus. The raised MDA levels indicate the oxidative stress and the decreased Vitamin $\mathrm{C}$ levels indicate the reduced antioxidant 
status in diabetic retinopathy. Hence, for the early detection and prevention of diabetic retinopathy, it is advisable to estimate the oxidative stress markers. The diet of diabetic patients should contain a recommended dietary allowance of vitamins to allow the non-enzymatic as well as the enzymatic antioxidant systems to respond to oxidative stress, which is observed among the diabetic changes.

\section{References}

1. Wild S, Roglic G, Green A, Sicree R, King H. Global prevalence of diabetes: Estimates for the year 2000 and projections for 2030. Diabetes Care 2004;27:1047-53.

2. Prevention of Blindness from Diabetic Retinopathy. Report of a WHO Consultation, Geneva; November, 2005.

3. Kumari S, Panda S, Mangraj M, Mandal MK, Mahapatra PC. Plasma MDA and antioxidant vitamins in diabetic retinopathy. Ind J Clin Biochem 2008;23(2):158-62.

4. Soliman GZA. Blood lipid per oxidation (superoxide dismutase, malondialdehyde, glutathione) levels in Egyptian type 2 diabetic patients. Singapore Med J 2008; 49(2):129-136.

5. Gupta.S, Ambade.A. Prevalence of diabetic retinopathy and influencing factors amongst type 2 diabetics from central India. Int. J. Diab. Dev. Countries 2004; 24:75-78.

6. Yau JWY, Rogers SL, Kawasaki R. Global prevalence and major risk factors of diabetic retinopathy. Diabetes Care 2012; 35:556-64.

7. The International Expert Committee. International Expert Committee report on the role of the A1C assay in the diagnosis of diabetes. Diabetes Care 2009; 32:1327-34.

8. Verma M, Alam R, Mobin M. Review on Malondialdehyde and Superoxide dismutase levels in patients of Type 2 Diabetes Mellitus with Retinopathy and without Retinopathy. Int. J. Life. Sci. Res.2015; 1(2): 52-57.

9. Tripathi P, Verma MK, Tripathi SS, Singh SP. Comparative Study of
Malondialdehyde and Vitamin C in Type 2 Diabetes Mellitus and Non Diabetic Individuals. Int. J. Life. Sci. Scientific. Res.2016; 2(1): 31-36.

10. Wu L, Fernandez-Loaiza P, Sauma J, Hernandez-Bogantes E, Masis M. Classification of diabetic retinopathy and diabetic macular edema. World J Diabetes. 2013;4(6):290-4.

11. Gupta S, Ambade A. Prevalence of diabetic retinopathy and influencing factors amongst type 2 diabetics from central India. Int. J. Diab. Dev. Countries 2004; 24:75-78.

12. Kurtul N, Bakan E, Hiilya A, Baykal. Leukocyte lipid peroxidation, superoxide dismutase and the catalase activities of type 2 diabetic patients with retinopathy. Acta Medica2005;48(1):35-38.

13. Lyons TJ, Jenkins AJ, Zheng D, Lackland DT, McGee D, Garvey WT, Klein RL. et al. Diabetic retinopathy and serum lipoprotein subclasses inthe DCCT/EDIC cohort. Invest Ophthalmol Vis Sci 2004; 45:91018.

14. Klein R, Klein BE, Moss SE, Davis MD, Demets DL. Glycosylated hemoglobin predicts the incidence and the progression of Diabetic retinopathy. J Am Med Assoc 1988; 260:2864-71.

15. Rosen P, Nawroth PP, King G, Moller W, Tritschler HJ, Packer 1, et al. The role of oxidative stress in the onset and progression of diabetes and its complications. Diabetes Metab Res Rev 2001; 17:189-212.

16. Philips M, Cataneo RN , Cheema T, Greenberg J. Increased breath biomarkers of oxidative stress in diabetes mellitus. Clinica Chimica Acta 2004; 344:189-194.

17. Unger J. Reducing oxidative stress in patients with Type 2 Diabetes Mellitus: A preliminary care cell to action. Insulin 2008; 3:176-184.

18. Wolff SP, Dean RT. Glucose auto oxidation and protein modification potential role of 'auto oxidative glycosylation' in diabetes. Biochem J 1987; 245:245-50.

19. Murat Y, Ayhan K, Nuri C, Füsun T, Ersel D, lhan Y, Göksun A, MetinA.The effects of vitamin $\mathrm{E}$ on oxidative stress and the metabolic status in Diabetes mellitus. Turkiye Klinkleri J Endocrin2004; 2:200205.

20. Kumari S, Panda S, Mangaraj M ,Mandal MK, Mahapatra PC. Plasma MDA and antioxidant vitamins in Diabetic Retinopathy. Indian J Clin Biochem2008; 23:158-162.

Copyright: (C) the author(s), 2020. It is an open-access article distributed under the terms of the Creative Commons Attribution License (CC BY 4.0), which permits authors to retain ownership of the copyright for their content, and allow anyone to download, reuse, reprint, modify, distribute and/or copy the content as long as the original authors and source are cited.

How to cite this article: Gahlawat R, Saksena DN. Study on Glycemic Status and Oxidative Stress Level in Patients of Type 2 Diabetes Mellitus With and Without Retinopathy: A Teaching Hospital Based Study. Asian J. Med. Res. 2020;9(1):OT01-OT04.

DOI: dx.doi.org/10.47009/ajmr.2020.9.1.OT1 